\section{SAT0247 EFFICACY AND SAFETY OF MODIFIED-RELEASE PREDNISONE IN MANAGING MODERATE ACTIVITY SYSTEMIC LUPUS ERYTHEMATOUS DURING PREGNANCY: AN IMPLEMENTED CASE-CONTROL STUDY}

M. Meroni ${ }^{1,2}$, V. Ramoni ${ }^{3}$, M. Limonta ${ }^{2}$, M. Cutolo ${ }^{1} .{ }^{1}$ Research Laboratory and Academic Division of Clinical Rheumatology, Internal Medicine Dept., University of Genova, Genova; ${ }^{2}$ Rheumatology Unit, ASST Papa Giovanni XXIII, Bergamo ${ }^{3}$ Division of Rheumatology, IRCCS San Matteo Hospital Foundation, University of Pavia, Pavia, Italy

Background: Systemic lupus erythematosus (SLE) primarily affects women of childbearing age. Despite the overall favorable outcome, pregnancy represents a challenge for both patients and clinicians. Since the complications rate is linked to the disease activity, the achievement of remission is recommended before pregnancy. Prednisone represents a cornerstone in SLE management and is safely used, at low doses ( $<7.5 \mathrm{mg}$ daily), during pregnancy. Modifiedrelease prednisone (MRP) optimize corticosteroid treatment strategy in rheumatic diseases, thanks to its capability of respect the physiological cortisol circadian secretion. MRP has been approved from FDA in SLE treatment, but no data are available regarding its administration during pregnancy.

Objectives: We aimed to investigate whether this drug is safe and effective as the immediate release prednisone (IRP) in SLE pregnant patients.

Methods: We retrospectively evaluated 9 female patients, fulfilling the ACR criteria for SLE, consulting our Centers in a 4-years observational range. All of them, thanks to a stable disease (not requiring treatment regimen modifications within 12 months), experienced a successful pregnancy during the observation. All the cases were taking low-dose MRP ( 5 to $7.5 \mathrm{mg} /$ daily) as a baseline treatment, from at least 6 months. They were matched to 9 controls, defined as SLE patients with the same age and duration of disease, taking the same prednisone dose, from at least 6 months, in the IR formulation. Overall pregnancy outcome features: SLE disease activity (calculated at least once during pregnancy, SLEPDAI) and at baseline/post-partum (SLEDAI) score; patient's global assessment (VAS) at baseline, during pregnancy and in postpartum $(\mathrm{mm})$; need of treatment changes throughout pregnancy and at postpartum (\%) were assessed. Homogeneity tests, percentages and scores comparison were run out by non-parametric statistical analysis.

Results: Mean MRP age group was $26 \pm 7$; disease duration, $4 \pm 8$ years; IR one, respectively, $28 \pm 6$ and $3 \pm 9$ (both, $p=n s$ ). SLEDAl at baseline was $1 \pm 0.1$ among MPR and $1 \pm 0.3$ among IR women; SLEPDAI, $1 \pm 0.9$ and $2 \pm 0.2$ (both, $\mathrm{p}=\mathrm{ns})$. No major perinatal complications were detected. Preterm births, cesarean section rates, newborns weight and APGAR scores did not differ between the two subpopulations (all, $p=n s$ ). SLEDAl assessed at postpartum was $2.8 \pm 0.6$ in MRP subjects and $3.4 \pm 0.4$ in IR $(p<0.05)$. Patients VAS (MRP vs IR) was $3 \pm 0.4$ and $2 \pm 09$ at baseline $(p=n s) ; 2 \pm 0.6$ and $4 \pm 0.7$ during pregnancy $(p<0.05)$ and $3 \pm 0.3$ and $4 \pm 0.9$ at postpartum $(p<0.05)$. Regarding treatment regimen changes (add-on strategy), the observed rates involved 1/9 (MRP) and 5/9 (IR) women during the observational gap (pregnancy+postpartum) $(p<0.001)$. Results synthesis is reported in Table I.

\begin{tabular}{|llll|}
\hline & Cases MRP & P value & Controls IRP \\
\hline Age (years \pm months) & $26 \pm 52$ & $\mathrm{~ns}$ & $28 \pm 43$ \\
\hline Disease duration (yearstmonths) & $4 \pm 8$ & $\mathrm{~ns}$ & $3 \pm 9$ \\
\hline PDN dose baseline (mg) & $5 \pm 2.5$ & $\mathrm{~ns}$ & $5 \pm 2.5$ \\
\hline SLEDAl baseline & $1 \pm 0.1$ & $\mathrm{~ns}$ & $1 \pm 0.3$ \\
\hline VAS baseline (mm) & $20 \pm 6$ & $\mathrm{~ns}$ & $21 \pm 5$ \\
\hline PDN dose pregnancy (mg) & $5 \pm 2.5$ & $\mathrm{p}<0.05$ & $7.5 \pm 2.5$ \\
\hline SLEPDAl & $1 \pm 0.9$ & $\mathrm{p}<0.05$ & $3 \pm 0.2$ \\
\hline VAS pregnancy (mm) & $41 \pm 8$ & $\mathrm{p}<0.05$ & $23 \pm 7$ \\
\hline Gestation (weeks days) & $38 \pm 5$ & $\mathrm{~ns}$ & $37 \pm 6$ \\
\hline Child weight (g) & $3230 \pm 420$ & $\mathrm{~ns}$ & $3120 \pm 390$ \\
\hline APGAR & $9 \pm 1$ & $\mathrm{~ns}$ & $9 \pm 0$ \\
\hline PDN dose postpartum (mg) & $2.5 \pm 2.5$ & $\mathrm{p}<0.05$ & $5 \pm 2.5$ \\
\hline PDN need to change (\%) & 11 & $\mathrm{p}<0.001$ & 56 \\
\hline SLEDAl postpartum & $2.8 \pm 0.6$ & $\mathrm{p}<0.05$ & $3.4 \pm 0.4$ \\
\hline VAS postpartum (mm) & $50 \pm 4$ & $\mathrm{p}<0.05$ & $71 \pm 6$ \\
\hline
\end{tabular}

Conclusions: Activity (SLEDAI) score was significantly higher at postpartum and treatment had to be increased in IR patients, in comparison to the MRP, to manage SLE. VAS, conversely, was significantly higher among IR, both during pregnancy and postpartum. No major perinatal side effects were observed during the study; minor and expected complications rates did not differ between the two subpopulations. Despite the limited number of subjects, MRP treatment seems to be as safe, but more effective, in comparison to the standard IR one, during pregnancy of SLE-affected women.

Disclosure of Interest: None declared

DOI: 10.1136/annrheumdis-2017-eular.3135 SAT0248 BASELINE CLASI-DAMAGE IS A MAJOR PREDICTOR OF SKIN
RESPONSE TO BELIMUMAB IN A MULTICENTRIC COHORT OF SLE PATIENTS

M. Gatto ${ }^{1}$, L. laccarino ${ }^{1}$, L. Andreoli ${ }^{2}$, F. Conti ${ }^{3}$, R. De Angelis ${ }^{4}$, S. De Vita ${ }^{5}$, L. Emmi ${ }^{6}$, R. Gerli ${ }^{7}$, M. Govoni ${ }^{8}$, M. Mosca ${ }^{9}$, C. Salvarani ${ }^{10}$, A. Tincani ${ }^{2}$,

A. Doria ${ }^{1} .{ }^{1}$ Unit of Rheumatology, University of Padova, Padova; ${ }^{2}$ Rheumatology and Clinical Immunology Unit, Spedali Civili and University of Brescia, Brescia;

${ }^{3}$ University LA Sapienza, Rome; ${ }^{4}$ Rheumatology Unit, Univerisity of Marche, lesi; ${ }^{5}$ University of Udine, Udine; ${ }^{6}$ AOU Careggi, Florence; ${ }^{7}$ Rheumatology Unit, University of Perugia, Perugia; ${ }^{8}$ Unit of Rheumatology, University of Ferrara, Ferrara; ${ }^{9}$ Unit of Rheumatology, University of Pisa, Pisa; ${ }^{10}$ Rheumatology Unit, Arcispedale S Maria Nuova - IRCCS, Reggio Emilia, Italy

Background: Belimumab is used to treat several systemic lupus erythematosus (SLE) manifestations and predictors of response are advisable in clinical practice. Objectives: To explore the effects of belimumab treatment on skin involvement in SLE patients in a real-life setting.

Methods: SLE patients treated with belimumab $(10 \mathrm{mg} / \mathrm{kg}$ day $0,14,28$ and then every 28 days), as add-on therapy in 11 Italian cohorts were prospectively followed-up for 24 months. Skin involvement was measured by the CLASla score (Cutaneous Cutaneous LE Disease Area and Severity Index Activity) at baseline and every six months until month 24. Damage was measured by CLASI-Damage (CLASId). Response was defined as CLASla $<2$ at measured timepoints.

The following variables were tested to determine baseline predictors of response at 12, 18 and 24 months: gender, age, disease duration, SLE activity index 2000 (SLEDAI-2K) $\geq 10$, prednisone dose $>7.5 \mathrm{mg} /$ day, baseline CLASla and CLASId, concomitant immunesuppressants (yes/no) and number of previous immunesuppressants. Statistical analysis was performed with SPSS 22.0 software.

Results: 188 patients were studied, among whom $48(25.5 \%)$ had skin involvement as the leading feature for belimumab therapy. Mean follow-up period was $17.5 \pm 10.96$ months. Thirty-eight patients completed a 6 -month follow-up; 27 completed a 12-month follow-up; 19 completed a 18-month follow-up and 15 completed a 24-month follow-up. Fourteen patients discontinued due to adverse events $(7 / 14,50 \%)$, lack of efficacy $(4 / 14,28.5 \%)$ or other causes $(3 / 14,21.4 \%)$. CLASla, daily prednisone dosage and SLEDAI-2K significantly decreased during the follow-up $(p<0.001)$. CLASla $<2$ was achieved by $25 / 38$ patients $(65.7 \%)$ at 6 months, $19 / 27(70 \%)$ at 12 months, $11 / 19(58 \%)$ at 18 months and 10/15 (67\%) at 24 months. A lower baseline CLASla was associated with CLASla response at 18 and 24 months (responders vs. non-responders: $3.4 \pm 2.4$ vs. $9.5 \pm 5.6 ; 3.5 \pm 2.5$ vs. $11.2 \pm 6.5 ; p<0.005$ for both), while a lower baseline CLASId was associated with CLASla response at each timepoint (responders vs. non-responders: at 12 months: $0.6 \pm 0.9$ vs. $2.4 \pm 2.5$; at 18 months: $0.5 \pm 0.8$ vs. $2.5 \pm 2.3$; at 24 months: $0.6 \pm 0.8$ vs. $3.25 \pm 2.7 ; p=0.01$ for all). Multivariate analysis was only performed at 12 months due to low patient number at 24 months. CLASId was the only independent negative predictor of response (OR 0.52, $p=0.05$ ). Notably, CLASId remained stable during the follow-up.

Conclusions: Belimumab use in cutaneous involvement is associated with reduced activity in skin lesions and hindrance of skin damage. Early use of belimumab before damage is established is likely to be associated with a better response.

Acknowledgements: dr. Margherita Zen, dr. Maddalena Larosa, dr. Francesca Ometto, dr. Mara Felicetti for helping in data collection and statistical analysis Disclosure of Interest: None declared

DOI: 10.1136/annrheumdis-2017-eular.6461

\section{SAT0249 SAFETY, PHARMACOKINETICS, PHARMACODYNAMICS AND INHIBITION OF T-CELL DEPENDENT ANTIBODY RESPONSE (TDAR) WITH MEDI4920, A NOVEL, ENGINEERED CD40 LIGAND (CD40L) ANTAGONIST: RESULTS OF A FIRST-TIME- IN-HUMAN STUDY}

M.G. Albulescu ${ }^{1}$, M. Gunsior ${ }^{2}$, J. Li ${ }^{3}$, J. Bush ${ }^{4}$, A. Godwood ${ }^{1}$, R. Miday ${ }^{2}$, E. Grant ${ }^{5}$, D. Howe ${ }^{1}$, R. Faggioni ${ }^{3}$, L. Roskos ${ }^{2}{ }^{1}$ Medlmmune, Cambridge, United Kingdom; ${ }^{2}$ Medlmmune, Gaithersburg; ${ }^{3}$ Medlmmune, Mountain View, United States; ${ }^{4}$ Covance Clinical Research Unit Ltd, Leeds, United Kingdom; ${ }^{5}$ Gilead Sciences Inc, Foster City, United States

Background: The CD40/CD40L pathway is involved in the T-cell-dependent activation of $B$ cells, which subsequently produce autoantibodies and inflammatory mediators that contribute to autoimmune disease pathology. MEDI4920 is an engineered fusion protein and antagonist of CD4OL that lacks the fragment crystallisable $(\mathrm{Fc})$ domain thought to be involved in thromboembolic events (TEs) previously reported with anti-CD40L agents containing an Fc domain.

Objectives: The primary objective of this Phase I, randomised, double-blind, placebo-controlled, single-ascending dose study was to evaluate the safety and tolerability of MEDI4920 in healthy subjects. Secondary objectives were to characterize T-cell-dependent antibody response (TDAR) to keyhole limpet hemocyanin (KLH) neoantigen, pharmacokinetics (PK), and anti-drug antibodies (ADAs) to MEDI4920.

Methods: Fifty-six healthy adult male subjects, aged 19-49 years, received a single intravenous dose of either MEDI4920 $(3,10,30,100,300,1000$ or $3000 \mathrm{mg})$ or placebo. TDAR inhibition was analysed by measuring serum concentrations of 